\title{
Re: Schweitzer T, Bohm H, Meyer-Marcotty P, Collmann H, Ernestus RI, Krauss J (2012) Avoiding CT scans in children with single-suture craniosynostosis. Childs Nerv Syst 28: 1077-1082
}

\author{
Paul Steinbok
}

Received: 12 October 2012 / Accepted: 23 October 2012 / Published online: 29 December 2012

(C) Springer-Verlag Berlin Heidelberg 2012

\begin{abstract}
Dear Editor:
I wish to complement Dr. Schweitzer and his colleagues on the report of their experience with the management of children with single-suture craniosynostosis [2]. I strongly agree with the authors that CT scans are rarely necessary for evaluation of these single-suture craniosynostosis. In fact, in 2006 we reported our series of isolated sagittal synostosis and showed that it was possible to accurately diagnose the condition based on clinical findings only [1]. Like the authors, we were concerned about the potential risks of radiation from a CT scan, especially in infancy. We concluded then that "Accurate diagnosis of isolated sagittal synostosis can be made clinically, and operative correction can proceed without a need for radiological investigations, unless the clinical features are not completely typical. This approach could result in significantly reduced radiation exposure for the developing brain and could provide economic benefits to health care providers."
\end{abstract}

We stand by these conclusions and at BC Children's Hospital in Vancouver we continue to avoid CT scans in almost all cases of isolated single-suture craniosynostosis.

We are pleased that others, such as Schweitzer and colleagues are doing the same. We hope that others will follow their lead.

Paul Steinbok

\section{References}

1. Agrawal D, Steinbok P, Cochrane DD (2006) Diagnosis of isolated sagittal synostosis: are radiographic studies necessary? Childs Nerv Syst 22:375-378

2. Schweitzer T, Bohm H, Meyer-Marcotty P, Collmann H, Ernestus RI, Krauss J (2012) Avoiding CT scans in children with singlesuture craniosynostosis. Childs Nerv Syst 28:1077-1082
P. Steinbok $(\bowtie)$

British Columbia Childrens Hospital,

Vancouver, BC, Canada

e-mail: psteinbok@cw.bc.ca 